\title{
Far-Field Errors Due to Random Noise in Cylindrical Near-Field Measurements
}

\author{
Jordi Romeu, Student Member, IEEE, Luis Jofre, Member, IEEE, and Angel Cardama, Member, IEEE
}

\begin{abstract}
A full characterization of the far-field noise obtained from cylindrical near- to far-field transformation, for a white Gaussian, space stationary, near-field noise is derived. A possible source for such noise is the receiver additive noise. The noise characterization is done by obtaining the autocorrelation of the far-field noise, which is shown to be easily computed during the transformation process. Even for this simple case, the far-field noise has complex behavior dependent of the measurement probe. Once the statistical properties of the far-field noise are determined, it is possible to compute upper and lower bounds for the radiation pattern for a given probability. These bounds define a strip within the radiation pattern is found with the desired probability. This may be used as part of a complete near-field error analysis of a particular cylindrical near-field facility.
\end{abstract}

\section{INTRODUCTION}

$\mathrm{N}$ EAR-FIELD antenna pattern measurement has become a widely employed technique over the past years. As the far-field pattern is obtained after processing the near-field data, it is difficult to foresee the effect of a given near-field error on the far-field pattern. Thus far, expressions are available [1], [2] that predict the effect of random errors for the planar case. These expressions are not valid for the cylindrical case, due to the different nature of the transformation process.

The far-field noise is a stochastic process, and for each measurement that is performed a new realization of the process is obtained. Therefore, it is not possible to specify the far-field error in terms of a deterministic value, but rather it has to be looked as a random variable whose distribution function has to be determined. This approach to the problem allows the computation of bounds for the error for a given probability, that is a value that it is not exceeded for a chosen probability. These bounds offer a better understanding of the effects of random errors in the far-field pattern. Moreover, it will be shown that for a white Gaussian, space stationary, near-field noise, the far field is a Gaussian, nonstationary in elevation, and colored in azimuth process. Thus, a parameter such as the far-field signal-to-noise ratio, although being a quite appealing simple parameter, hides in its simplicity a complex phenomenon.

The paper is organized as follows. In Section II the autocorrelation of the far-field noise for a white Gaussian

Manuscript received April 16, 1991; revised August 22, 1991.

The authors are with the Department of Signal theory and Communications, Telecommunication Engineering School of Barcelona, Polytechnic University of Catalonia, 08080 Barcelona, Spain.

IEEE Log Number 9105280. near-field noise is derived. In Section III is shown how to evaluate the autocorrelation from the near-field measurement, and its application to predict the error bounds. Section IV shows a simulated as well as experimental verification.

\section{FAR-Field NoISE Autocorrelation}

The theory of the cylindrical near- to far-field transform has been well developed elsewhere [3]-[5]. In this section, we will employ those expressions necessary to develop the error formulation. At the moment, only the case of a complex white Gaussian, space stationary, with zero mean, and a variance $\sigma^{2}$ near-field noise is considered. This model assumes that all random errors are of Gaussian nature, an assumption that it is true for certain sources of error like receiver floor noise, but incorrect for other sources as quantization errors. Nevertheless, the Gaussian model is accurate enough for most measurement situations where lack of dynamic range is the fundamental limitation.

The cylindrical near- to far-field transformation is based on obtaining the cylindrical modal coefficients of the fields radiated by the antenna under test (AUT). Once the coefficients are known, the asymptotic expression of the far fields as a function of the modal coefficients is employed to compute the AUT far field. Two measurements corresponding to two different measurement probes are required to obtain the modal expansion of the AUT fields. From a theoretical point of view very few constraints are placed on the choice of the measurement probes, but in practice the probes are chosen so each one responds basically to one orthogonal polarization.

In the following analysis the statistical characteristics of the far-field noise due to the transformation of the near-field noise are found. The analysis is performed by considering near-field data containing only noise, and applying the cylindrical near- to far-field transform to this data.

Let $n_{n f}\left(z_{i}, \phi_{l}\right)$ be the measured near field, white Gaussian noise over the cylindrical measurement surface. The measurement grid is defined by the sample points $z_{i}$ and $\phi_{l}$. The vertical and azimuthal sampling spacing is $\Delta z$ and $\Delta \phi$, and they are assumed to be constant. The number of measured rings is $N_{z}$, and the number of measured points per rings is $N_{\phi}$.

The first step in the transformation process is the Fourier transform of the near field. Let $k_{z}$ and $n$ be the variables in the transformed domain. The discrete Fourier transform (DFT) of the near-field noise is

$$
\tilde{n}_{n f}\left(k_{z}, n\right)=\frac{\Delta z \Delta \phi}{2 \pi} \sum_{i}^{N_{z}} \sum_{l}^{N_{\phi}} n_{n f}\left(z_{i}, \phi_{l}\right) e^{j k_{z} z_{i}} e^{-j n \phi_{l}} .
$$


The autocorrelation of the DFT of the noise is [6]

$$
\begin{aligned}
R_{N}(p, q) & =\left(\frac{\Delta \phi \Delta z}{2 \pi}\right)^{2} \sigma^{2} \sum_{i}^{N_{z}} \sum_{l}^{N_{\phi}} e^{-j p \phi_{i}} e^{j p z_{i}} \\
& =N_{z} N_{\phi} \sigma^{2}\left(\frac{\Delta \phi \Delta z}{2 \pi}\right)^{2} \delta(p, q) .
\end{aligned}
$$

This autocorrelation function corresponds to a white Gaussian noise, because the spectral power density, that is defined as the Fourier transform of the autocorrelation, is flat. The cylindrical modal coefficients corresponding to the expansion of the near-field noise in cylindrical waves are related to the near-field Fourier transform as

$$
\begin{aligned}
& a_{n}\left(k_{z}\right)=-\frac{k \eta}{16 \pi^{2} k_{\rho}^{2}} \\
& \cdot \frac{\tilde{n}_{n f}^{h}\left(k_{z}, n\right) b_{n}^{(2)}\left(-k_{z}\right)-\tilde{n}_{n f}^{v}\left(k_{z}, n\right) b_{n}^{(1)}\left(-k_{z}\right)}{a_{n}^{(1)}\left(-k_{z}\right) b_{n}^{(2)}\left(-k_{z}\right)-a_{n}^{(2)}\left(-k_{z}\right) b_{n}^{(1)}\left(-k_{z}\right)} \\
& b_{n}\left(k_{z}\right)=-\frac{k \eta}{16 \pi^{2} k_{\rho}^{2}} \\
& \cdot \frac{\tilde{n}_{n f}^{v}\left(k_{z}, n\right) a_{n}^{(1)}\left(-k_{z}\right)-\tilde{n}_{n f}^{h}\left(k_{z}, n\right) b_{n}^{(2)}\left(-k_{z}\right)}{a_{n}^{(1)}\left(-k_{z}\right) b_{n}^{(2)}\left(-k_{z}\right)-a_{n}^{(2)}\left(-k_{z}\right) b_{n}^{(1)}\left(-k_{z}\right)}
\end{aligned}
$$

where $a_{n}^{(i)}\left(k_{z}\right)$ and $b_{n}^{(i)}\left(k_{z}\right)$ are related to the modal coefficients of the measurement probe, and can be computed as shown in [3] or [4], and $\tilde{n}_{n f}^{h}$ and $\tilde{n}_{n f}^{v}$ are the DFT of the noise in the horizontal and vertical measurement. $\eta$ is the medium impedance, $k=\omega \sqrt{(\mu \epsilon)}$, and $k_{\rho}=\sqrt{k^{2}-k_{z}^{2}}$. In this case the modal coefficients are stochastic processes, and assuming that $\tilde{n}_{n f}^{h}$ and $\tilde{n}_{n f}^{v}$ are statistically independent, the autocorrelation of the coefficients is

$$
\begin{aligned}
R_{a_{n}}(p, q) & {\left[\frac{k \eta}{16 \pi^{2} k_{\rho}^{2}}\right]^{2} } \\
& \cdot \frac{\left|b_{n}^{(2)}\left(-k_{z}\right)\right|^{2}+\left|b_{n}^{(1)}\left(-k_{z}\right)\right|^{2}}{\left|a_{n}^{(1)}\left(-k_{z}\right) b_{n}^{(2)}\left(-k_{z}\right)-a_{n}^{(2)}\left(-k_{z}\right) b_{n}^{(1)}\left(-k_{z}\right)\right|^{2}} \\
& \cdot \sigma^{2} N_{z} N_{\phi}\left(\frac{\Delta \phi \Delta z}{2 \pi}\right)^{2} \delta(p, q)
\end{aligned}
$$

and

$$
\begin{aligned}
R_{b_{n}}(p, q) & \\
= & {\left[\frac{k \eta}{16 \pi^{2} k_{\rho}^{2}}\right]^{2} } \\
& \cdot \frac{\left|a_{n}^{(1)}\left(-k_{z}\right)\right|^{2}+\left|a_{n}^{(2)}\left(-k_{z}\right)\right|^{2}}{\left|a_{n}^{(1)}\left(-k_{z}\right) b_{n}^{(2)}\left(-k_{z}\right)-a_{n}^{(2)}\left(-k_{z}\right) b_{n}^{(1)}\left(-k_{z}\right)\right|^{2}} \\
& \cdot \sigma^{2} N_{z} N_{\phi}\left(\frac{\Delta \phi \Delta z}{2 \pi}\right)^{2} \delta(p, q) .
\end{aligned}
$$

Now $a_{n}\left(k_{z}\right)$ and $b_{n}\left(k_{z}\right)$ are Gaussian random variables with zero mean, but are not stationary as their autocorrelation is a function of $n$ and $k_{z}$. Each far-field component is obtained as [3]

$$
\begin{aligned}
& E_{\theta}\left(k_{z}, \phi\right)=-2 k \sin \theta \sum_{n}^{N_{\phi}} j^{n+1} b_{n}\left(k_{z}\right) e^{j n \phi} \\
& E_{\phi}\left(k_{z}, \phi\right)=j 2 k \sin \theta \sum_{n}^{N_{\phi}} j^{n+1} a_{n}\left(k_{z}\right) e^{j n \phi}
\end{aligned}
$$

with

$$
\begin{aligned}
& k_{z}=k \cos \theta \\
& k_{\rho}=k \sin \theta .
\end{aligned}
$$

The autocorrelation of the $\theta$ far-field noise component is

$$
\begin{aligned}
R_{n_{\theta}}(\tau, \xi)= & E\left\{n_{\theta}\left(k_{z}+\tau, \phi+\xi\right) n_{\theta}^{*}\left(k_{z}, \phi\right)\right\} \\
= & (2 k \sin \theta)^{2} \sum_{m} \sum_{n} E\left\{b_{n}\left(k_{z}+\tau\right) b_{m}^{*}\left(k_{z}\right)\right\} \\
& \cdot j^{n+1} j^{-(m+1)} e^{j n(\phi+\xi)} e^{-j m \theta} \\
= & (2 k \sin \theta)^{2} \sum_{m} \sum_{n} R_{b_{n}}(n-m, \tau) \\
& \cdot j^{(n-m)} e^{j(n-m) \phi} e^{j n \xi}
\end{aligned}
$$

as $R_{b_{n}}(n-m, \tau)$ is zero if $n \neq m$

$$
\begin{gathered}
R_{n_{\theta}}(\tau, \xi) \\
=\sigma^{2}\left[\frac{k \eta}{8 \pi^{2} k_{\rho}}\right]^{2} N_{z} N_{\phi}\left(\frac{\Delta \phi \Delta z}{2 \pi}\right)^{2} \\
\sum_{n} \frac{\left|a_{n n}^{(1)}\left(-k_{z}\right)\right|^{2}+\left|a_{n}^{(2)}\left(-k_{z}\right)\right|^{2}}{\left|a_{n}^{(1)}\left(-k_{z}\right) b_{n}^{(2)}\left(-k_{z}\right)-a_{n}^{(2)}\left(-k_{z}\right) b_{n}^{(1)}\left(-k_{z}\right)\right|^{2}} \\
\cdot e^{j n \xi} \delta(\tau) .
\end{gathered}
$$

The autocorrelation for the $\phi$ component is derived in an analogous way. The far-field noise is white Gaussian nonstationary in $k z$, because the autocorrelation is a function of $k z$, and stationary and colored in $\phi$ because the autocorrelation is nonzero for $\zeta \neq 0$. The far-field noise variance for each polarization is

$$
\begin{gathered}
\sigma_{f f \theta}^{2}\left(k_{z}, \phi\right) \\
=R_{n \theta}(0,0) \\
=\sigma^{2}\left[\frac{k \eta}{8 \pi^{2} k_{\rho}}\right]^{2} N_{z} N_{\phi}\left(\frac{\Delta \phi \Delta z}{2 \pi}\right)^{2} \\
\sum_{n} \frac{\left|a_{n}^{(1)}\left(-k_{z}\right)\right|^{2}+\left|a_{n}^{(2)}\left(-k_{z}\right)\right|^{2}}{\left|a_{n}^{(1)}\left(-k_{z}\right) b_{n}^{(2)}\left(-k_{z}\right)-a_{n}^{(2)}\left(-k_{z}\right) b_{n}^{(1)}\left(-k_{z}\right)\right|^{2}}
\end{gathered}
$$

and

$$
\begin{aligned}
& \sigma_{f f \phi}^{2}\left(k_{z}, \phi\right) \\
& \quad=R_{n \phi}(0,0)=\sigma^{2}\left[\frac{k \eta}{8 \pi^{2} k_{\rho}}\right]^{2} N_{z} N_{\phi}\left(\frac{\Delta \phi \Delta z}{2 \pi}\right)^{2} \\
& \sum_{n} \frac{\left|b_{n}^{(1)}\left(-k_{z}\right)\right|^{2}+\left|b_{n}^{(2)}\left(-k_{z}\right)\right|^{2}}{\left|a_{n}^{(1)}\left(-k_{z}\right) b_{n}^{(2)}\left(-k_{z}\right)-a_{n}^{(2)}\left(-k_{z}\right) b_{n}^{(1)}\left(-k_{z}\right)\right|^{2}} .
\end{aligned}
$$


From the above expressions, the effect of an additive Gaussian white noise in the near field is an additive Gaussian noise with variance proportional to the near-field noise variance and function of $k z$. Moreover, the variance depends on the probe cylindrical coefficients (radiation pattern), so the effects of the noise will be dependent on the probe and in general different for each polarization.

\section{FAR-FIELD ERROR BOUNDS} form

The noise-contaminated radiation pattern will be of the

$$
\overrightarrow{\tilde{E}}\left(k_{z}, \phi\right)=\vec{E}\left(k_{z}, \phi\right)+\vec{n}\left(k_{z}, \phi\right)
$$

where $\vec{n}\left(k_{z}, \phi\right)$ is a Gaussian noise with variance $\sigma_{\mathrm{ff}}^{2}\left(k_{z}\right)$ given by (10) and (11). The far-field magnitude is for a given polarization

$$
\begin{aligned}
|\tilde{E}| & =|\mathscr{R}(E+n)+j \mathscr{J}(E+n)| \\
& =\left(\mathscr{R}(E+n)^{2}+\mathscr{J}(E+n)^{2}\right)^{1 / 2} \\
& =\left(X^{2}+Y^{2}\right)^{1 / 2}
\end{aligned}
$$

where $X$ and $Y$ are the real and imaginary parts of $E\left(k_{z}\right.$, $\phi)+n\left(k_{z}, \phi\right) . X$ and $Y$ are Gaussian random variables with mean value $\mathscr{R}(E)$ and $\mathscr{J}(E)$, and variance $\sigma_{\mathrm{re}}^{2}$ and $\sigma_{\mathrm{im}}^{2}$, respectively, and

$$
\sigma_{\mathrm{re}}^{2}=\sigma_{\mathrm{im}}^{2}=\frac{1}{2} \sigma_{\mathrm{ff}}^{2} .
$$

$|\tilde{E}|$ is a random variable with a Rice probability density function (pdf) given by [7]

$$
f(|\tilde{E}|)=\frac{|\tilde{E}|}{\sigma_{\text {re }}^{2}} e^{-\frac{|\tilde{E}|^{2}+|E|^{2}}{2 \sigma_{\text {re }}^{2}}} I_{0}\left(\frac{|\tilde{E}||E|}{\sigma_{\text {re }}^{2}}\right)
$$

where $I_{0}$ is the modified Bessel function of order zero. When $|E|^{2} / \sigma_{\mathrm{re}}^{2} \gg 1$, the Rice pdf can be approximated by a Gaussian pdf 'with mean value $|E|$, on the other hand if $|E|^{2} / \sigma_{\text {re }}^{2} \ll 1$ we have a Rayleigh pdf with mean value $\sigma_{\mathrm{re}} \sqrt{(\pi / 2)}$. Notice that in the latter case the mean value of the obtained far-field pattern is independent of the actual value of the radiation pattern $|E|$.

Knowing the pdf of the module of the far field, an upper and lower bound $M$ and $m$ for $|\tilde{E}|$ can be calculated by

$$
\begin{gathered}
P(|\tilde{E}| \leq M)=\int_{0}^{M} f(|\tilde{E}|) d|\tilde{E}|=p \\
P(|\tilde{E}|>m)=1-\int_{0}^{m} f(|\tilde{E}|) d|\tilde{E}|=q
\end{gathered}
$$

where $p$ and $q$ are the probabilities to exceed the bound $M$ and not to exceed $m$, respectively. The probability that the far field is between $M$ and $m$ is

$$
P(m<|\tilde{E}| \leq M)=\int_{m}^{M} f(|\tilde{E}|) d|\tilde{E}|=p+q-1 \text {. }
$$

To compute the far-field bounds, it is required to evaluate the far-field noise variance. This can be easily accomplished during the transformation process. It should be noted that the probe correction coefficients needed to evaluate (10) and (11) are also computed to produce the near- to far-field transform. Nevertheless, one unknown remains left to be found as the far field noise variance is proportional to the near-field noise variance $\sigma^{2}$.

The near-field variance can be estimated from the near-field measurement by taking into account the bandlimited properties of the AUT fields in the spectral domain $n-k_{z}$. The AUT field representation is limited to the visible domain defined by

$$
\left(\frac{k_{z}}{k}\right)^{2}+\left(\frac{n}{k a}\right)^{2}<1
$$

where $a$ is the radius of the minimum cylinder that encloses the AUT. Considering (2), the near-field noise variance can be estimated as

$$
\sigma^{2}=\frac{1}{N_{z} N_{\phi}}\left(\frac{2 \pi}{\Delta_{z} \Delta_{\phi}}\right)^{2} \frac{1}{N} \times \sum_{k_{z}, n \in \text { evanescent }}^{N}\left|\tilde{E}_{\mathrm{nf}}\left(k_{z}, n\right)\right|^{2}
$$

where $\tilde{E}_{\mathrm{nf}}$ is the DFT of AUT near-field plus the white Gaussian near-field noise $n_{\mathrm{nf}}(z, \phi)$, and the summation is extended over the evanescent region of the spectral domain. The variance is computed as the ensemble average of the noise power. This estimation procedure assumes that the noise in the spectral domain is stationary, which in this case is true.

It should be noted that the pdf of the noise perturbed far field (15) is a function of the far-field noise variance $\sigma_{\mathrm{ff}}^{2}$ and the unperturbed far-field magnitude $|E|$. In order to compute the bounds $m$ and $M$ given by (16) and (17) it is necessary to know the unperturbed value of the far-field pattern. Obviously this is not available from a noise-contaminated near-field measurement, but usually a certain $a$ priori knowledge of the AUT radiation pattern is available.

It must be noted that the far-field signal to noise ratio defined as

$$
(\mathrm{S} / \mathrm{N})_{\mathrm{ff}}=\frac{\left|E_{\mathrm{ff}}^{\max }\right|^{2}}{\sigma_{\mathrm{ff}}^{2}}
$$

can be easily computed after the transformation process.

\section{VERIFICATION}

Numerical and experimental verification of the above formulation has been done. To verify (10) and (11), the far-field noise variance has been estimated by performing a high number of near- to far-field transforms, with only noise as input. The far-field noise variance is estimated as

$$
\sigma_{\mathrm{ff}}^{2}\left(k_{z}, \phi\right)=\frac{1}{N} \sum_{i=1}^{N}\left[n_{\mathrm{ff} i}\left(k_{z}, \phi\right)\right]^{2}
$$

where $n_{\mathrm{ffi}}$ is the $i$ th transformed far-field noise, and $N$ is the number of transformations. Figs. 1 and 2 show the result after 1000 transformations, compared to the evaluation of 


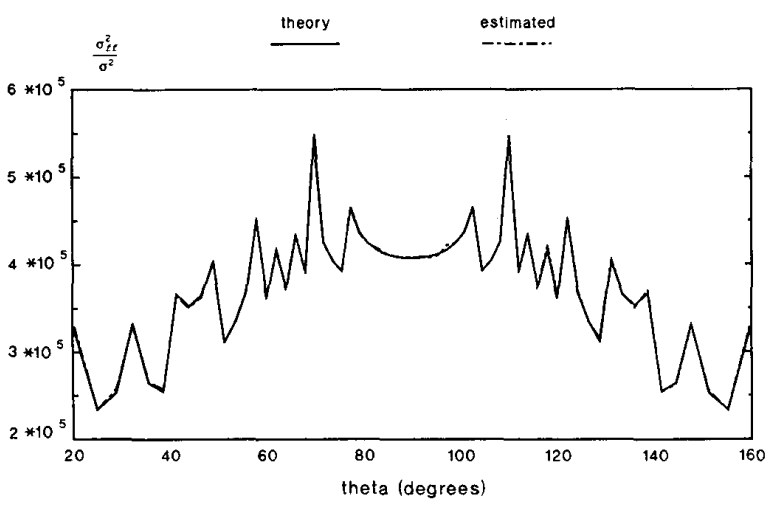

(a)

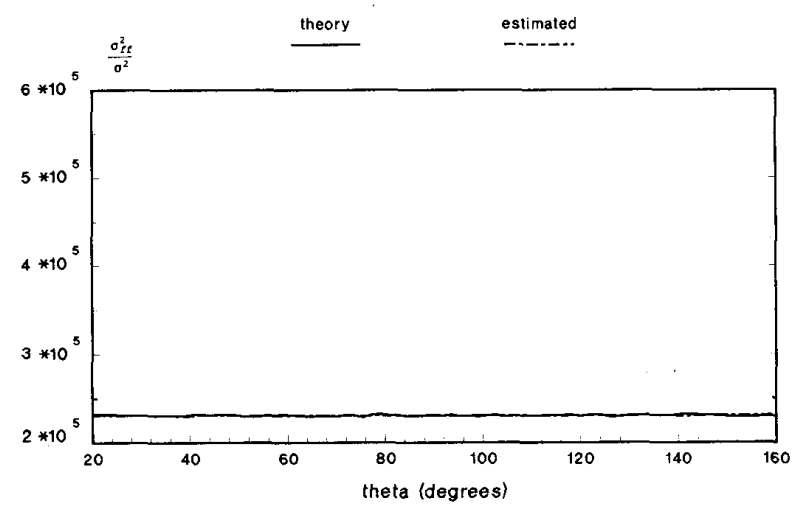

(b)

Fig. 1. Ratio between the far-field noise variance and the near-field noise variance for an ideal magnetic probe. The measurement parameters are case 1 of Table I. (a) Theta polarization, magnetic probe, $f=3 \mathrm{GHz}$. (b) Phi polarization, magnetic probe, $f=3 \mathrm{GHz}$.

expressions 10 and 11. Two different cases and specifics are shown in Table I. The probe employed in case 2 is a rotationally symmetric probe that is rotated $90^{\circ}$ along its axis. Due to the symmetry of the probe the far-field variance is the same for both polarizations. It is important to notice that the far-field variance actually depends on the measurement probe and the elevation angle.

Fig. 3 shows the numerically simulated far field for a low sidelobe array and the predicted upper and lower bounds defining an $80 \%$ probability strip when the near-field noise is $40 \mathrm{~dB}$. Fig. 4 shows the far field when the near-field $S / N$ is actually $40 \mathrm{~dB}$. Fig. 5 shows the radiation pattern for a $50 \mathrm{~dB}$ $\mathrm{S} / \mathrm{N}$ ratio and the computed bounds. From Figs. 4 and 5 it is concluded that in this case a $40 \mathrm{~dB} \mathrm{~S} / \mathrm{N}$ ratio practically smears the sidelobes, while for $50 \mathrm{~dB}$ an error of approximately $\pm 2 \mathrm{~dB}$ is expected in the first sidelobes.

The experimental verification has been done by perturbing a near-field measurement with a near-field $S / N$ greater than $50 \mathrm{~dB}$. The cylindrical near-field measurement range is described in [8]. The far-field pattern obtained from the initial measurement has been considered an error-free pattern, and used as unperturbed far field to compute the error bounds when the near-field $\mathrm{S} / \mathrm{N}$ is smaller than $50 \mathrm{~dB}$. The near-field data has been perturbed by adding noise to achieve the

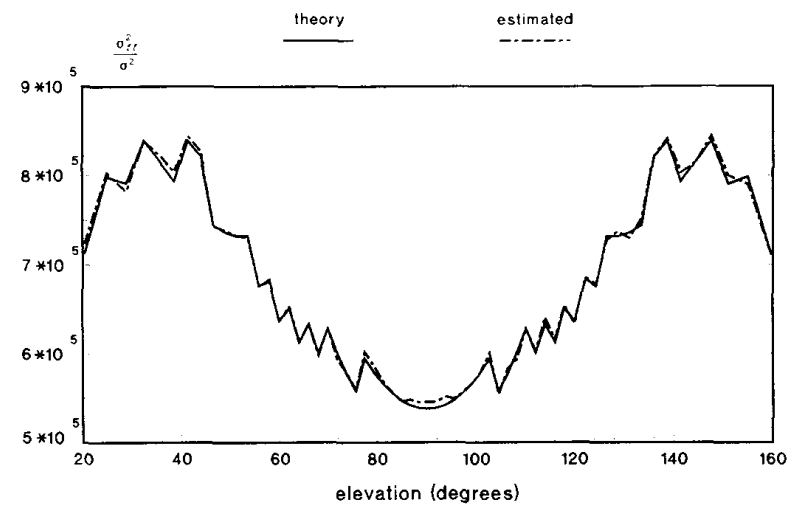

(a)

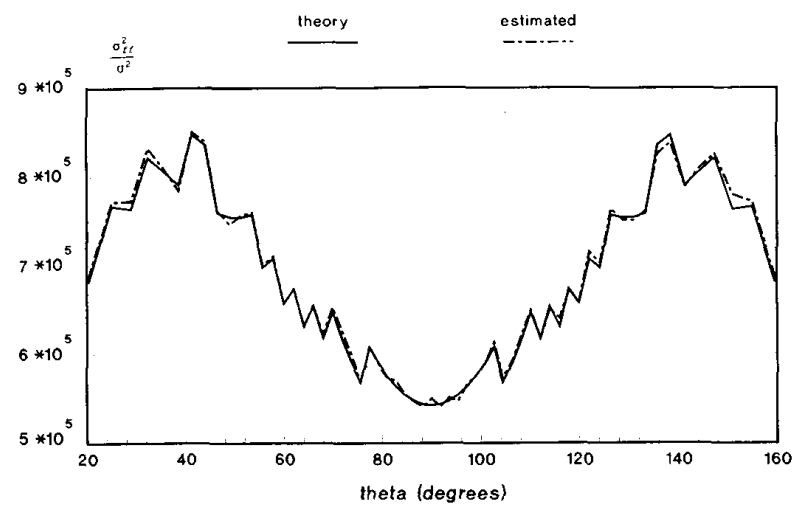

(b)

Fig. 2. Ratio between the far-field noise variance and the near-field noise variance for real probe. The measurement parameters are case 2 of Table $I$. (a) Theta polarization, real probe, $f=10 \mathrm{GHz}$. (b) Phi polarization, real probe, $f=10 \mathrm{GHz}$.

TABLE I

Measurement Parameters Employed in the Evaluation OF Figs. 1 AND 2

\begin{tabular}{lccccccc}
\hline & $\begin{array}{c}f \\
\end{array}$ & & & & & $\rho_{0}$ & \\
& $(\mathrm{GHz})$ & $N_{z}$ & $N_{\phi}$ & $\frac{\Delta z}{\lambda}$ & $\frac{\Delta \phi}{(2 \pi / N \phi)}$ & $(\mathrm{m})$ & Probe \\
\hline Case 1 & 3 & 64 & 64 & 0.5 & 1 & 0.51 & magnetic \\
Case 2 & 10 & 64 & 64 & 0.5 & 1 & 0.15 & real \\
\hline
\end{tabular}

desired $\mathrm{S} / \mathrm{N}$. In Table II is shown the near-field $\mathrm{S} / \mathrm{N}$ and the estimated value obtained as described in Section III. The error in the estimation is in general lower than $1 \mathrm{~dB}$ and increases for large and small values of the $S / N$. For large values of the $\mathrm{S} / \mathrm{N}$ ratio the noise estimation is more subject to error, because the assumption that there is a null contribution of the field in the evanescent region it is not completely true in the near-field zone, and as the noise variance becomes smaller the error is greater. On the other hand, for low $\mathrm{S} / \mathrm{N}$ the value of the maximum field becomes more corrupted by the noise, resulting in a wrong estimation of the $S / N$.

Fig. 6 shows the error bounds and the error in the radiation pattern for a $40 \mathrm{~dB} \mathrm{~S} / \mathrm{N}$. The results show how a good prediction of the error due to random noise can be accomplished. 


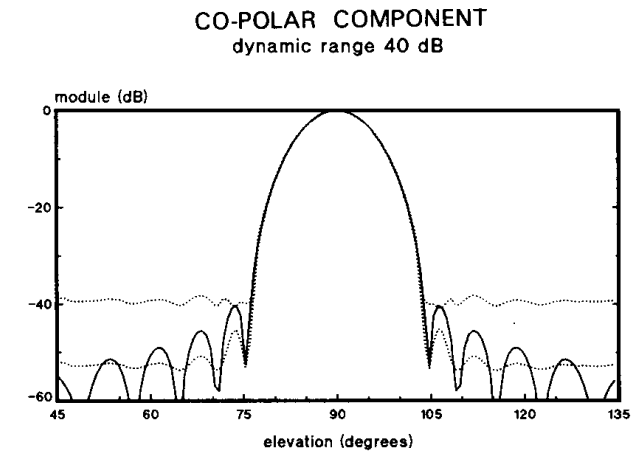

(a)

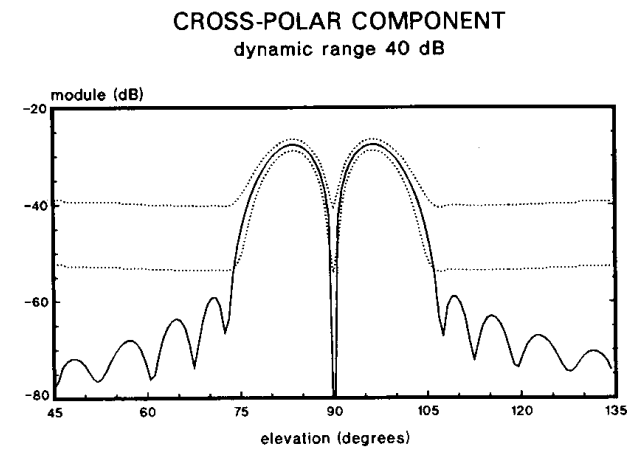

(b)

Fig. 3. In solid lines co-polar (a) and cross-polar (b) radiation pattern for a low sidelobe antenna and in dotted lines $90 \%$ bounds for a $40 \mathrm{~dB}$ near-field $\mathrm{S} / \mathrm{N}$ ratio. The bounds define an $80 \%$ probability strip.

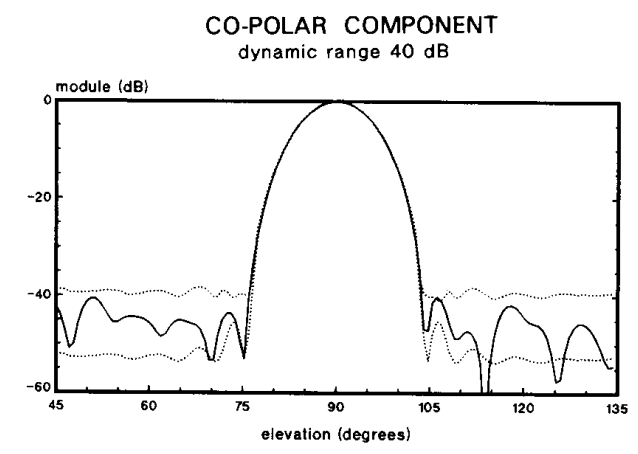

(a)

CROSS-POLAR COMPONENT dynamic range $40 \mathrm{~dB}$

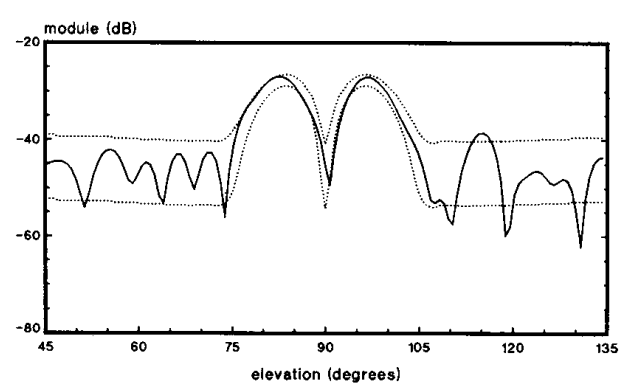

(b)

Fig. 4. Computed radiation pattern when the near-field $S / N$ ratio is $40 \mathrm{~dB}$.
CO-POLAR COMPONENT

dynamic range $50 \mathrm{~dB}$

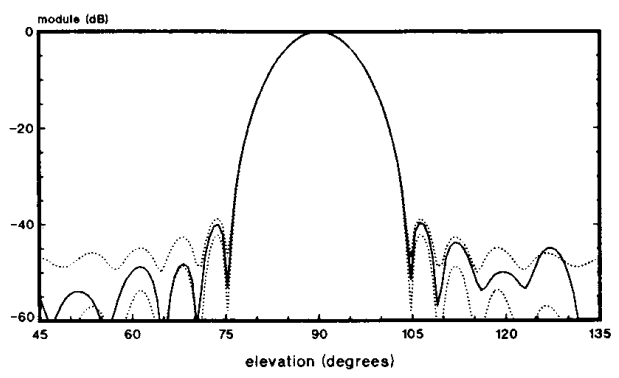

(a)

CROSS-POLAR COMPONENT

dynamic range $50 \mathrm{~dB}$

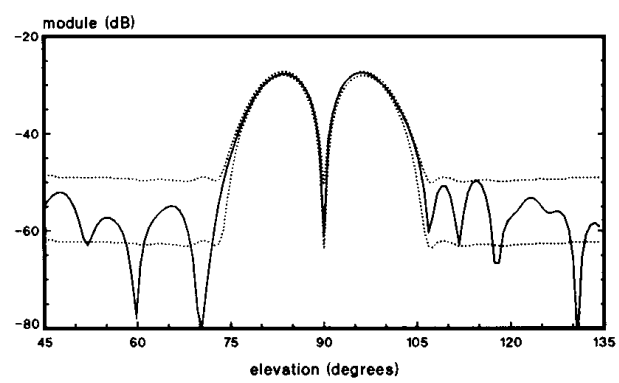

(b)

Fig. 5. Computed radiation pattern when the near-field $S / N$ ratio is $50 \mathrm{~dB}$ and the computed bounds defining an $80 \%$ probability strip.

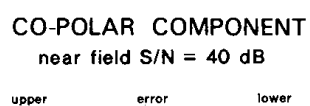

upper $\quad$ error $\quad$ lower

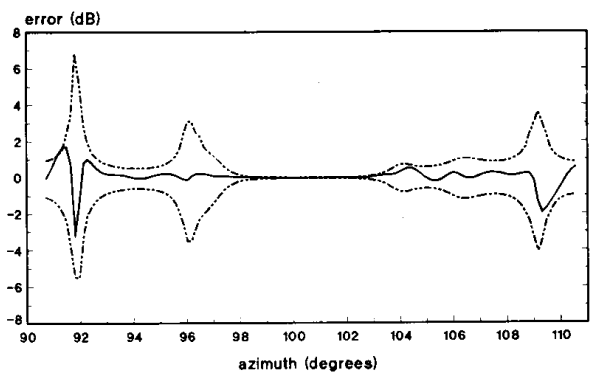

Fig. 6. Error for a real measurement for a $40 \mathrm{~dB}$ near-field $\mathrm{S} / \mathrm{N}$ ratio and computed bounds for a $90 \%$ probability, defining an $80 \%$ probability strip.

TABLE II

Near-Field S/N Ratio and Estimation from the Near-Field MEasurement

\begin{tabular}{ccc}
\hline $\begin{array}{c}\text { Near-Field S/N } \\
(\mathrm{dB})\end{array}$ & $\begin{array}{c}\text { Estimated } \mathrm{S} / \mathrm{N} \\
(\mathrm{dB})\end{array}$ & $\begin{array}{c}\text { Error } \\
(\mathrm{dB})\end{array}$ \\
\hline 15 & 16.78 & 1.78 \\
20 & 20.57 & 0.57 \\
25 & 25.26 & 0.26 \\
30 & 30.01 & 0.01 \\
35 & 34.98 & -0.02 \\
40 & 39.71 & -0.29 \\
45 & 44.02 & -0.98 \\
\hline
\end{tabular}




\section{CONCLUSION}

In this paper an expression for the far-field noise autocorrelation for a near-field Gaussian random noise is derived. The expression considers the effect of the measurement probe and it is shown that the probe plays an important role in the determination of the far-field noise variance. It is important to notice that the far-field noise variance is proportional to the number of sample points, and proportional to the square of the sampling space. So in a real measurement situation, where the dynamic range requirements may be critical, it is important to choose these parameters correctly. This expression can be easily evaluated during the near- to far-field transformation. Once the far-field noise is characterized, it is possible to predict the effect of the noise on the radiation pattern. This requires a certain knowledge of the unperturbed radiation pattern. As the noise is of random nature, the far-field error is given in terms of an error with a chosen probability.

\section{REFERENCES}

[1] A. C. Newel and C. F. Stubenrauch, "Effect of random errors in planar near-field measurement," IEEE Trans. Antennas Propagat., vol. 36, pp. 769-773, June 1988.

[2] J. B. Hoffman and K. R. Grimm, "Far-field uncertainty due to random near-field measurement error," IEEE Trans. Antennas Propagat., vol. 36, pp. 774-780, June 1988.

[3] W. M. Leach and D. T. Paris, "Probe compensated near-field measurements on a cylinder," IEEE Trans. Antennas Propagat., vol. AP-21, pp. 435-446, July 1973.

[4] G. V. Borgiotti, "Integral equation formulation for probe corrected far-field reconstruction from measurements on a cylinder," IEEE Trans. Antennas Propagat., vol. AP-26, pp. 572-578, July 1978.

[5] A. D. Yaghjian, "Near-field antenna measurements on a cylinder surface: A source scattering matrix formulation," Nat. Bur. Stand., NBS Tech. Note 696, July 1977.

[6] A. Papoulis, Probability, Random Variables, and Stochastic Processes. New York: McGraw-Hill, 1965.

[7] D. O. North, "An analysis of the factors which determine signal/noise discrimination in pulsed-carrier systems," Proc. IEEE, vol. 51, pp. 1016-1027, July 1963.

[8] J. Romeu et al., "A cylindrical near-field test facility," Microwave Eng. Europe, pp. 25-31, Sept./Oct. 1990.

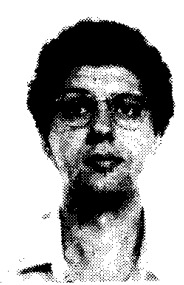

Jordi Romeu (S'88) was born in Barcelona, Spain, in 1962. He received the Ingeniero and Doctor Ingeniero degrees in telecommunication engineering, both from the Polytechnic University of Catalonia (UPC), Barcelona, Spain, in 1986 and 1991, respectively.

In 1985 he joined the Antenna-Microwave-Radar group of the Signal Theory and Communications Department there. Currently he is Associate Professor at UPC, where he is engaged in research in tics and microwave imaging. antenna near-field measurements, antenna diagnos-

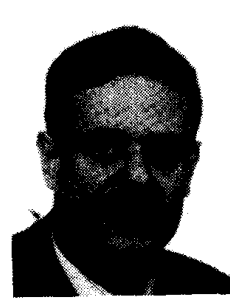

Luis Jofre (S'79-M'83) was born in Mataró, Spain, in 1956. He received the Ingeniero and Doctor Ingeniero degrees in telecommunication engineering, both from the Polytechnic University of Catalonia (UPC), Barcelona, Spain, in 1978 and 1982, respectively.

In 1978 he was Research Assistant in the Electrophysics group at UPC, where he worked on the analysis and the near-field measurement of antennas. In 1981 he joined the Ecole Supérieure d'Electricité in Paris, where he was involved in microwave imaging techniques for biomedical applications. During the period 1986-1987 he was a Visiting Fulbright Scholar at the Georgia Institute of Technology, Atlanta, working on antenna measurement and electromagnetic imaging. He is currently Professor and Director of the Telecommunication Engineering School at UPC where he is engaged in research on antennas and electromagnetic scattering and imaging, both numerical and experimental aspects.

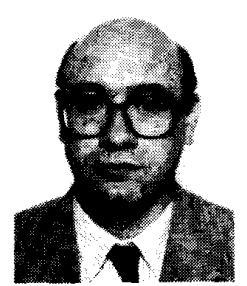

Angel Cardama (S'67-M'73) was born in Santiago, Spain, on May 13, 1944. He received Ingeniero de Telecomunicación degree from the Polytechnic University of Madrid, Madrid, Spain, in 1968, and the Sc.M. and Ph.D. degrees in electrical engineering from Brown University, Providence, RI, in 1970 and 1973, respectively.

In 1972 he joined the faculty of the E.T.S.I. de telecomunicación at the Polytechnic University of Catalonia, Barcelona, Spain, where he holds the position of Professor. His research interests range from propagation in optical fibers, high frequency aperture and array antennas, and near-field antenna scanning systems to the design of microwave imaging systems and radar antennas. 